\section{$\underset{\text { hommes }}{\text { \& migrations }}$}

\section{Hommes \& migrations}

Revue française de référence sur les dynamiques

migratoires

\section{$1308 \mid 2014$}

Les Paris des migrants

\title{
Peggy Derder, Idées reçues sur les générations issues de
}

l'immigration

Paris, Le Cavalier Bleu, 2014, $18 €$.

\section{Catherine Guilyardi}

\section{(2) OpenEdition}

\section{Journals}

Édition électronique

URL : http://journals.openedition.org/hommesmigrations/3037

DOI : 10.4000/hommesmigrations.3037

ISSN : 2262-3353

Éditeur

Musée national de l'histoire de l'immigration

Édition imprimée

Date de publication : 1 octobre 2014

Pagination : 194

ISBN : 978-2-919040-29-2

ISSN : 1142-852X

Référence électronique

Catherine Guilyardi, «Peggy Derder, Idées reçues sur les générations issues de l'immigration », Hommes \& migrations [En ligne], 1308 | 2014, mis en ligne le 10 juillet 2015, consulté le 24 septembre 2020. URL : http://journals.openedition.org/hommesmigrations/3037 ; DOl : https://doi.org/10.4000/ hommesmigrations.3037 


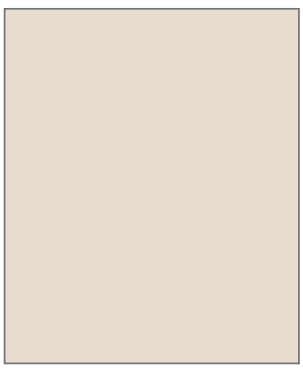

Jean-Paul

\section{Gourévitch}

Les Migrations

pour les nuls

éd. First, 2014

454 p., 22,95€

Dans la collection "Pour les nuls", cet ouvrage propose de retracer en 454 pages les migrations d'hier, d'aujourd'hui et de demain, de définir les termes, de montrer les flux et d'expliquer la géopolitique des déplacements, ainsi que la quantité et la "qualité" des migrations. Jean-Paul Gourévitch, auteur prolifique (son dernier ouvrage porte sur la littérature jeunesse), est présenté sur la couverture comme "consultant international sur l'Afrique et les migrations" et se présente lui-même, dans l'introduction, comme ayant "le souci constant de faire le point sur des sujets mal connus ou sensibles et de lutter contre la désinformation d'où qu'elle vienne". II s'est fait connaître, sur le sujet, pour ses livres La Croisade islamiste et L'immigration ça coûte ou ça rapporte? (Larousse, 2009), cités en référence par Marine Le Pen.

La lecture de ce gros livre, proposé par Gourévitch aux éditions First, met mal à l'aise. Alors que l'auteur préconise la prudence en matière de chiffres, "compte tenu des incertitudes statistiques (...), les chiffres qui seront présentés dans les chapitres suivants sont à consommer avec modération", il se lance dans la longue démonstration d'un "surcoût" de l'immigration de 8,9 milliards d'euros par an pour le budget de la France, puis tente de chiffrer le retour sur investissement de la rénovation des quartiers sensibles ou des subventions aux associations antiracistes. Pour le "citoyen responsable" que ce livre voudrait aider à "construire sa propre opinion en connaissance de cause", il est difficile de comprendre ce qu'il faut retenir tant il y a de chiffres, de faits, de graphiques ou d'anecdotes, sans contextualisation ni sources, dans la plupart des cas. Ses sources, l'auteur les donne peut-être dans sa longue liste de remerciements où se trouvent, pêlemêle, le Musée de l'immigration [sic], la Cour des comptes, le Gisti, la Maison des esclaves de Gorée, l'Ascé ou "I'exFasild" devenu... l'Acsé.

Pourquoi taire, par exemple, la source de ce chiffre étonnant de 594697 euros, coût estimé de la famille de Leonarda "aux contribuables" selon des "analyses de la presse" ? Le calcul vient du Figaro et il est facile à trouver sur le site de l'association Contribuables associés, à laquelle Gourevitch contribue régulièrement. Quel sens cela a-t-il, ensuite, que l'auteur propose son propre chiffre, "plus modeste", prenant en compte le "ravitaillement en cigarettes par le comité des sans-papiers"?

Dans une interview au site de Riposte laïque, Gourevitch disait : "En matière d'immigration, par exemple, la quasitotalité des experts se sont trompés7." Dans Les Migrations pour les nuls, "il montre, aussi, sa méfiance vis-à-vis des médias, taxant certains d'"officiels" ou d'immigrationnistes", sans guillemets. C'est "un ouvrage sérieux sans être pesant", affirme l'auteur, qui introduit des "récréations" telles un "bêtisier des migrations" (annexe B), liste de cita- 
tions pour "donner à réfléchir". Premier par ordre alphabétique, les rappeurs AMER commencent avec "J'ai envie de dégainer sur ces faces de craie". Wihtol de Wenden (Catherine) finit avec : "II est erroné de dire 'Si vous ne voulez pas de Marocains, prenez leurs tomates', car on a à la fois les tomates et les Marocains". Dans cette liste, tout est placé au même niveau, des propos de la chercheuse, sans aucun contexte, à la petite annonce de N'guéyé (Mamadou), un "mage vaudou", qui propose la "réparation de votre PC par télépathie".

Le manque de références, l'inexactitude des termes et les amalgames entre islam et islamisme ou étranger et descendant d'immigrés, sont encore plus gênants dans le chapitre intitulé "Dix débats de fond pour comprendre les migrations". À la question "La délinquance est-elle le corollaire de l'immigration?", l'auteur propose des arguments pour et contre. Premier argument pour : "Les statistiques carcérales sont éloquentes. II y a surreprésentation des étrangers et plus particulièrement des étrangers musulmans dans les prisons. La preuve par les nombreux imams qui y professent." Premier argument contre: "Il ne faut pas confondre la petite délinquance, visible au quotidien et dont on peut admettre qu'elle est majoritairement d'origine étrangère, avec la grande délinquance, dans laquelle étrangers et immigrés sont peu ou pas du tout représentés."

Comment un tel ouvrage a-t-il pu paraître ? La maison d'édition, qui ne répond pas à nos appels, a simplement déclaré au Monde : "L'auteur est un spécialiste du sujet, il est consultant international." Gourévitch explique, dans une interview au NouvelObs.fr, que l'ou- vrage a été peu corrigé, faute d'éditeur spécialisé. La présence de coquilles grossières, comme "crote" au lieu de croate ou "Essel" pour Stéphane Hessel, suggère que le livre n'a pas été relu du tout. Dans un débat si polémique, où nombre de questions sont difficiles à trancher, faute de statistiques ou parce que les migrations recouvrent des réalités complexes, pourquoi avoir choisi un auteur critiqué pour ses positions sur le sujet, estampillé par certains à l'extrême droite ? Le livre est introuvable sur le site www.pourlesnuls.fr mais on le trouve sur Amazon, qui précisait, fin septembre, que "les clients ayant acheté cet article [avaient] également acheté": Comprendre l'Empire d'Alain Soral, Pour en finir avec l'idélogie antiraciste de Paul-François Paoli et L'Allemagne disparait de Thilo Sarrazin, qui écrit : Soumise à des influences islamistes, autoritaires et anti-démocratiques, [la] communauté musulmane, dont le nombre croit très fortement, pourrait devenir une menace directe pour l'avenir du style de vie allemand."

La lecture des Migrations pour les nuls provoque, au mieux, une grande confusion sur un sujet complexe, au pire, l'impression que l'auteur veut faire passer ses idées sans l'afficher ouvertement.

Catherine Guilyardi

1. http://ripostelaique.com/jean-paul-gourevitch-auteur-de-la-croisade-islamiste-il-y-a-en-france-entre7-et-8-millions-de-musulmans.html 


\section{Ian Coller}

Une France arabe 1798-1831. Histoire des débuts de la diversité

Alma Éditeur, 2014, 381 p., $29 €$

En 1811, les immigrés égyptiens qui avaient suivi les troupes napoléoniennes vaincues, en 1801, ont enfin le droit de quitter Marseille, où ils furent cantonnés dix ans, et Melun, où se trouvaient leurs casernes, pour s'installer à Paris. L'Égypte pacifiée et stabilisée n'est plus un enjeu militaire et Napoléon souhaite utiliser ces immigrés éduqués (pour la plupart), afin d'entretenir de bons rapports diplomatiques avec le nouvel émir.

Dans les chapitres 3 et 4 de son livre au titre prudent "Une" France arabe, l'Australien Ian Coller retrace la topographie de ce Paris arabe d'avant la période coloniale, un "espace jamais nommé". Venu en France en 2003 pour faire cette "enquête" historique sur ce qu'il nomme "un monde englouti", le jeune historien retrouve des sources précieuses dans les archives de l'armée à Vincennes, des centaines de lettres adressées aux autorités françaises, dont beaucoup en arabe, écrites par ces premiers "Arabes" de France : Égyptiens, Libanais, Syriens, musulmans mais aussi chrétiens. Les deux chapitres développant leur présence dans la capitale, de 1810 jusqu'à la Restauration, dressent le portrait de plusieurs personnages et tentent, à partir de ces individus et de leur interaction avec leur entourage, de reconstruire le monde dans lequel ils évoluent. Ce sont souvent des personnages hauts en couleur, dissemblables - parfois en opposition ouverte -, mais réunis dans une même "communauté" (l'auteur se revendique de la définition de James Clifford). Ce sont, écrit l'auteur, "des existences disparates reliées par un espace dans lequel elles évoluent constitué de la langue arabe et de l'islam, et de l'adaptation de ces deux points communs entre ces individus à la France en pleine transformation elle-même", de la Révolution à l'expansion coloniale, et grâce au développement de l'industrie et du commerce.

En 1810, Paris est le cœur de l'Empire, considéré comme "la nouvelle Rome" et la "capitale des Lumières". Le jeune Champollion y fréquente quelques figures du Paris arabe, notamment Rufa'il Zakhur, prêtre syrien, traducteur personnel de Napoléon lors de sa campagne d'Égypte, nommé en exil professeur auxiliaire d'arabe vulgaire de l'École des langues orientales de Paris, créée en 1795. L'homme servira de modèle au peintre David pour son moine d'Orient au capuchon rouge, présent aux côtés de l'Empereur dans le Sacre de Napoléon.

L'iconographie de l'époque - gravures, lithographies et peintures - tient une place de choix dans l'ouvrage de lan Coller, montrant souvent ces Arabes de France sous les traits des pittoresques mamelouks qui rallièrent Napoléon et fuirent l'Égypte. Mais l'auteur montre aussi que la présence de ces hommes paraît normale dans les rues et les parcs parisiens. Plusieurs centaines d'Orien- 
taux se trouvent en France au début du XIXe siècle, peut-être un millier, selon l'auteur, et ils ne sont pas de passage, mais bien installés.

Ils arrivent progressivement à Paris et ne sont pas cantonnés à un seul quartier. Les moins favorisés (ceux qui recevaient la pension de l'État la plus faible) choisissent les alentours du Louvre, alors un quartier pauvre, à deux pas de l'obélisque de la Concorde, "le plus ancien monument de Paris", rappelle astucieusement lan Coller. Les plus aisés, soit parce qu'ils occupent - chose rare - un emploi, soit parce que la pension qu'ils reçoivent est élevée (due à leur importance sociale ou/et à celle de leur progéniture) peuvent vivre dans le $X V l^{e}$ arrondissement, comme par exemple Georges Aïdé, monté de Marseille en 1810, avec ses nombreux enfants. Aïdé est un "notable" de la communauté à Marseille, et tient à le rester à Paris. Une violente altercation avec un des membres de cette communauté disparate composée de lettrés, de religieux, d'anciens soldats ou de commerçants est consignée dans les lettres conservées dans les archives de l'armée, que l'historien a ressorties avec émotion des cartons. Ce différend entre Aïdé et Joubran Mehanna, visible aussi dans le registre de police 1806-1811, permet de comprendre le réseau de relations qu'entretenaient ces Arabes de Paris entre eux et avec les Français, ou tout du moins avec les autorités françaises. Les lettres sont adressées au ministre de la Guerre, chargé de régler la dispute survenue après le refus du plus aisé, Aïdé, d'aider le moins établi. Cet incident, nous explique Ian Col- ler, illustre la confrontation de deux attitudes opposées dans l'exil : "Aïdé se montrait outragé devant 'l'imagination exaltée' de Joubran, ce qui laisse entendre qu'il envisageait le comportement asocial de cet homme plus jeune que lui comme l'expression de son refus d'envisager le monde comme structuré par les coutumes, les normes propres à la communauté. Ce conflit d'identités, me semble-t-il, est crucial pour comprendre la violence de cette rencontre. Joubran semblait se vivre comme un Arabe ayant adopté l'identité parisienne." II se voulait un "citoyen cosmopolite d'un nouveau genre", explique l'historien, mais, dans le besoin, Joubran se retourne pourtant vers un des notables de cette communauté qu'il veut fuir. Cet incident marque aussi la frustration d'une communauté qui peine à s'établir dans un régime où les réseaux importent plus que le mérite.

Ce paysage parisien connaît une fin brutale en 1815, après le massacre à Marseille d'Égyptiens, ou de tous ceux dont le visage fait penser qu'ils le sont, en représailles à leur soutien à l'Empereur déchu et exilé après sa défaite de Waterloo, le 18 juin 1815. Cette "présence" arabe disparaît de la mémoire collective, le sujet de leur influence sur la société locale devenant un "éléphant dans le salon français". Leur disparition fait place, dans la construction de la mémoire, aux immigrés issus de la conquête de l'Algérie, de la colonisation, puis de la décolonisation. Dans ce livre passionnant, Ian Coller rappelle que ceux-ci ne furent pas les premiers Arabes à interagir avec la société française. C. G. 


\section{Peggy Derder}

Idées reçues sur les

générations issues

de l'immigration

Le Cavalier Bleu, 2014,

173 p., $18 €$

Peggy Derder, spécialiste d'histoire contemporaine et enseignante, responsable du départe-

ment pédagogie du Musée de l'histoire de l'immigration, répond à 16 idées reçues sur ceux qui, nés en France d'un ou deux parents ou grandsparents immigrés, se trouvent "figés dans un rapport d'extranéité (...) bien que le statut d'immigré ne soit pas héréditaire". Les descendants d'immigrés sont-ils en échec scolaire ou responsables de la délinquance ? Déracinés ? Refusent-ils de voter ? Subissent-ils davantage de discriminations que leurs parents? Plus souvent au chômage que le reste des actifs ? L'auteure, actuellement responsable de l'action éducative du Musée de l'histoire de l'immigration, se demande aussi si "les générations issues de l'immigration n'ont pas intégré le monde des arts et de la culture". Basé sur des études sociologiques et statistiques récentes, telle l'enquête $\mathrm{TeO}$ sur la diversité des populations en France menée par l'Ined et l'Insee en 20082009, ce livre s'attache à utiliser les termes exacts. Pourquoi qualifie-t-on toujours ces enfants de "jeunes" ? "La jeunesse ne s'éternis[e] pas! prévient Peggy Derder. La présence de descendants d'immigrés de plus en plus âgés composera le nouveau visage de la société française à l'avenir." En quelques pages seu- lement, elle répond à des idées simples qui méritent clarification, telle que "le regroupement familial est la première source d'immigration", ou plus complexes comme le supposé regain de religiosité ou un taux de délinquance supérieur chez les descendants de Maghrébins et d'Africains. Si elle parle de toutes les vagues d'immigration, elle ne noie pas le poisson, comme dans le chapitre sur l'attitude religieuse: "Autant poser la question sans détour, c'est bel et bien la place de l'islam dans la société (...) qui est considéré comme un problème." Son but, que l'on devine proche de celui du Musée d'histoire de l'immigration - "faire évoluer les regards et les mentalités sur l'immigration en France" -, ne l'empêche pas de confirmer certaines idées reçues. Le mariage forcé concerne en premier lieu les filles d'immigrés mais il s'agit d'une toute petite minorité et les générations suivantes sont peu touchées. Elle oppose aussi des sources comme les travaux du chercheur Hugues Lagrange (CNRS), qui affirme que, dans un contexte de relégation sociale, "les valeurs traditionnelles et familiales des pays d'origine sont plus prégnantes et pérennes", aux réponses de Patrick Simon et Vincent Tiberj (TeO), pour qui "ces musulmans plus religieux que leurs parents se trouvent dans toutes les catégories sociales ou niveaux d'éducation". Malgré la difficulté - et son refus de tracer un portrait global de ces "jeunes" issus de l'immigration, Peggy Derder nous montre que l'origine de leurs parentsn'estqu'un,etunseul,descritères de choix et de comportements pour les descendants d'immigrés.

C. G. 


\section{Kamel Daoud}

Meursault, contre-enquête

Actes Sud, 2014, 152 p., $19 €$

Et si Kamel Daoud était la face lumineuse d'une histoire dont Éric Zemmour, cet autre Algérien, juif berbère et indigène devenu français par un décret Crémieux par lui mal digéré, symboliserait la part d'ombre? La comparaison est osée. À chacun ses marottes et ses carottes. Mais enfin, le romancier et le polémiste médiagénique ont en commun l'histoire franco-algérienne et ses troubles post-traumatiques. L'un n'en sort pas quand l'autre ouvre un chemin de résilience. Comment? En revisitant Camus et son Étranger. En interrogeant deux trilogies, celle qui hante l'âme tourmentée des Algériens - CamusMeursault-l'Arabe - et l'autre qui hante la conscience troublée des Français l'indigène-l'Arabe-le musulman.

De quoi s'agit-il ? De donner une suite à L'Étranger, de rendre nom et histoire à cet "Arabe" invisible, muet, anonyme - "on ne tue pas un homme facilement quand il a un prénom" -, abandonné sur une plage d'Alger, le corps troué de balles. Daoud visite l'absence. Le silence. Le trou noir de la France en Algérie. II interroge le présent, celui de son pays. Le devenir, celui des siens.

La victime s'appelait Moussa Ould elAssasse. Un demi-siècle plus tard, dans un bar d'Oran, un esquif menacé par la superbe des buveurs d'eau, son frère raconte à un universitaire français cette histoire où "l'absurde, c'est mon frère et moi qui le portons sur le dos, pas l'autre". II y a là "un serveur kabyle à carrure de géant, un sourd-muet apparemment tuberculeux, un jeune universitaire à l'œeil sceptique et un vieux buveur de vin qui n'a aucune preuve de ce qu'il avance". $\mathrm{Au}$ centre du récit, enfle une quête irraisonnée de vengeance. Elle est portée par M'ma, la mère de Moussa, et l'injonction faite à Haroun, son dernier rejeton, de rechercher le corps de son frère, de venger sa mort et de tenter de faire valoir ses droits aux nouveaux maîtres du pays. Puis elle s'enfermera dans la folie, le silence et un deuil éternel. Haroun, lui, vivra écrasé par la culpabilité. Et ses morts.

Kamel Daoud écrit en français, dans une langue nerveuse. "Quand les colons s'enfuient, ils nous laissent souvent trois choses : des os, des routes et des mots ou des morts..." Cette langue est celle qu'utilise Haroun, non comme un "butin de guerre" mais "pour faire barrage au délire de sa mère". Une langue comme un ailleurs, comme une "solution aux dissonances de mon monde. Je voulais le traduire à M'ma, mon monde, et le rendre moins injuste en quelque sorte". La construction figure un tourbillon centripète où virevoltent la société coloniale et ses ressorts rouillés mais encore grinçants, les "indigestions" de l'indépendance: la voracité d'une "bête qui s'était nourrie de sept ans de guerre", les minarets écrasants, les ennuyeux vendredis, le corps des femmes réduit à "un péché ou une honte”, le désir, la rêverie, l'affolement des sens condamnés, l'ombre noire du passé et des morts sur la vie et l'amour. 
Dans cette mécanique ondulatoire, Kamel Daoud creuse et creuse jusqu'à atteindre le point nodal, matriciel, celui des violences vaines. À "ton Caïn a tué mon frère pour... rien" répond un autre crime, comme "un contrepoids à l'absurde". "La gratuité de la mort de Moussa était inadmissible", mais la vengeance, "frappée de la même nullité !", laissera le meurtrier prisonnier des "ténèbres".

C'est bien une "histoire de fous", "de morts gratuites", qui est racontée. "Com- ment prendre la vie au sérieux ensuite?" Partout "le crime compromet pour toujours l'amour et la possibilité d'aimer". Camus et Daoud convergent. Comme "L'heure du crime ne sonne pas en même temps pour tous les peuples", dixit Cioran en exergue de ce roman, chacun a rendez-vous avec l'histoire : aux Algériens de rédiger ce "traité de la digestion" de l'indépendance ; aux Français d'en finir avec ce qu'Alexis Jenni a appelé "la pourriture coloniale". Mustapha Harzoune

\section{Linda Lê}

\section{Par ailleurs (exils)}

Christian Bourgois, 2014, 163 P., $13 €$

L'exil, les exilés !, sont au cœur de la modernité, du métissage des consciences et du devenir planétaire. "Ces oiseaux de passage migrent d'un espace imaginaire à un autre, leur univers est bipolaire, leur âme flotte incertaine entre des étendues distinctes, entre l'Est et l'Ouest, entre le Nord et le sud. Ils ne subsistent en terre étrangère qu'en s'efforçant d'échapper à tout catalogage. Sans renier leurs origines, ils sont des hybrides, à cheval sur deux mondes (...). Ils font de leur appétit de savoir un moyen de s'affranchir d'un trop grand enracinement dans le sol natal qui contrarie le développement intellectuel. Ils sont les porte-drapeaux des inquiets et des inassouvis, dépourvus d'axe cen- tral, inaptes aux conquêtes, masquant tant bien que mal leurs manques, mais à leur manière, ils sont des agents de la subversion. (...) Déplacés, hétérodoxes, ils soulèvent des questions intempestives, telles que le non à la morale figée, à la dictature de la normalité." L'époque est et sera demain celle des réfugiés, des déplacements de populations, des migrations tous azimuts, des métissages et des inventions d'identités nouvelles. "Cioran prédisait même qu'elle serait celle du romantisme des apatrides : 'déjà se forme l'image d'un univers où plus personne n'aura droit de cité. (...) Dans tout citoyen d'aujourd'hui git un métèque futur." Linda Lê ouvre sa bibliothèque. II faut la suivre.

Qui sont-ils ces exilés par Linda Lê réunis dans ce recueil de textes, dans lesquels elle met en perspective les œuvres, les thèmes et ses auteurs de prédilection? Qui sont-elles ces figures non pas de l'exil mais des exils ? Il y a les déracinés, les migrants de toutes sortes et de tout acabit. Il y a les exilés de l'intérieur, 
dans leur propre pays. Ceux qui refusent de céder une once de leur liberté. Ils résistent ! Au colonialisme, au stalinisme ou au nazisme. Ici figurent aussi les rebelles aux "mystifications collectives", celles qui courent le monde. Enfin, il y a l'exil de l'artiste, seul, travaillé par "l'intranquillité" de la création et des veilles.

Pour parler de ces exils, Linda Lê a convoqué près de soixante écrivains. Autant de trajectoires biographiques, souvent sombres, et d'œuvres, qui conduisent à serpenter sur des chemins de résistance. Chacun est gratifié de sa notice, riche en commentaires et opportunes citations. Notices sensibles et indispen- sables pour mieux appréhender la marche, chaotique, du monde.

De quoi s'agit-il ? D’une longue réflexion sur la peur instinctive de l'Autre, sur la singularité de l'exilé, sur l'altérité comme un voyage vers soi-même, sur l'errance, le cheminement, l'instabilité, le contretemps, la fantaisie et la déraison, sur les métissages et les sang-mêlé, ces "abrégés de l'univers". Sur l'écriture aussi, comme nouveau lieu d'habitation. "L'artiste en rupture" serait "un visionnai$r e^{\prime \prime}$ et l'écrivain n'aurait ou ne devrait avoir "pour mission de ne s'adresser qu'à l'individu, de région profonde à région profonde".

M. H.

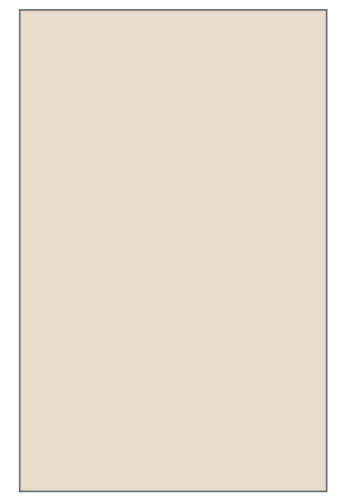

\section{Richard \\ Beraha (dir.)}

La Chine à Paris.

Enquête au cœur

d'un monde

méconnu

Robert Laffont, 2012, 308 p., $21 €$

La Chine à Paris présente une approche interdisciplinaire d'universitaires chinois et français, une étude de terrain fruit de dix années d'observation et d'actions associatives et s'ouvre avec le témoignage de Liwen Dong, en France depuis 2002 et membre fondateur de l'association Convergence.

La France compterait 450000 à 700000 personnes d'origine chinoise, voire $1 \mathrm{mil}-$ lion selon Pierre Picquart (L'Empire chi- nois, Favre, 2004). Les Chinois originaires du district de Wenzhou en représenteraient $70 \%$. Sachant que depuis une trentaine d'années la majorité sont entrés en France clandestinement, on mesure l'efficacité - et l'inanité - des politiques de maîtrise des flux migratoires.

$\mathrm{Au}$ lendemain de l'ouverture économique de la République populaire, les Wenzhous renouent avec une expérience qui remonte à plusieurs générations et qui a pu constituer, en France et dans les quelque 104 pays où ils se sont installés, autant de points d'appui. Commerces, restaurants, ateliers divers, import-export, immobilier, réseaux d'approvisionnement, de distribution, circulation de l'argent via dons, prêts, investissements, placements entre la Chine et les pays de résidence..., ce 
dynamisme repose sur une culture spécifique et des structures d'entraide sociales et familiales particulièrement fortes, "fascinantes", écrit Richard Beraha, qui précise : "Pour expliquer la migration Wenzhou, les facteurs économiques et sociaux liés à la région d'origine et à ceux du pays d'accueil ne sont pas suffisants. (...) Partir à l'étranger représente essentiellement un signe de distinction sociale, un habitus, qui se fonde sur une expérience collective de départ outre-mer depuis des générations et est facilité par les réseaux ainsi constitués."

Selon l'auteur, cette réussite commerciale ne doit rien à des groupes ni à des pratiques maffieuses. Reste que les "têtes de serpent" constituent "un des sujets les plus tabous sur lequel règne l'omerta". Cela posé, le livre montre que le succès de la diaspora chinoise repose sur une économie informelle, constituée d'un réseau dense d'entreprises privées et familiales, pratiquant prêts et dons en circuit fermé. Un "capitalisme familial" basé sur "la confiance". Entre Wenzhous, la parole suffit: "Tu me prêtes tant, je te rembourse tant et le contrat est scellé." L'appartenance à des cercles communs, les liens du sang, les réseaux de lignage ou la proximité villageoise garantissent que personne ne perdra la "face". Le système qui enjambe les frontières exige un vaste réseau familial et social qu'il convient d'élargir au fil du temps et au rythme des fêtes.

Car les cérémonies et festivités sont autant d'occasions de souder la communauté, de multiplier liens et alliances, de recevoir des dons ou d'emprunter. Tout est bon pour entretenir l'énergie de la réussite : une naissance, un décès, l'inauguration d'un commerce, une régularisation, un anniversaire... et surtout un mariage. L'institution, centrale, scelle moins l'union de deux cœurs enamourés que la réunion et le renforcement de deux réseaux. Comme le Wenzhou est aussi bouddhiste, taoïste, confucéen ou chrétien (10\% seraient protestants) et que l'œcuménisme festif s'étend aux fêtes du calendrier national, laïques ou catholiques, ou commerciales (Saint-Valentin), cela multiplie d'autant les occasions de réjouissances et d'entretenir les affaires. Cette réussite a sa part d'ombre : des conditions de travail dangereuses, une surexploitation qui s'apparente à de l'esclavage et ce jusqu'au remboursement de la dette aux passeurs (jusqu'à 20000 euros), des années de clandestinité, sans droits, dans la crainte de la police ou d'un employeur hostile, créant une "culture de la clandestinité" perverse et contreproductive pour la société.

Les Wenzhous sont longtemps restés "enfermés dans une Chine en France". Mais, avec les nouvelles générations, la multiplication des engagements associatifs et politiques, arrive le temps d' "une Chine de France". Ainsi, "derrière la capacité des Wenzhous à entreprendre gisent d'énormes potentialités pour notre pays". Le fier coq gaulois s'acoquinera-t-il avec le cochon chinois, symbole de richesse et de prospérité ? Car le dynamisme commercial des Chinois est impressionnant. L'investissement global des Chinois se situerait entre 3 et 7 milliards d'euros sur vingt ans. II conviendrait selon l'auteur d'ouvrir ce vaste et prometteur chantier, et fissa. 
Ne s'agit-il pas là de s'inspirer de l'art (chinois) des opportunités ?

Pour Richard Beraha, "les Chinois nous trouvent souvent trop bavards, trop curieux, comme si pour avancer dans le monde, nous avions sans cesse besoin de le décrire, de le conceptualiser, de le projeter pour le maitriser, alors qu'eux semblent l'affronter avec patience et défi, opportunisme et jeu, fatalité et résilience, dans l'attente d'un signe qui annoncera un vent favorable qu'ils saisiront alors avec impétuosité". Un tableau éclairant montre, à partir de plusieurs thèmes (langue, rapport au temps, stratégie, action, changement, efficacité, modes d'expression, le moi et l'autre, justice, relations et échanges, médecine...), les "écarts" (pour reprendre François Jullien) d'approche et de sensibilité entre l'Occident et la Chine. Ainsi, "la liberté, la vérité, l'humanisme individuel, la transparence et la sincérité, la fiabilité, la raison ou l'éthique" constituent les fondements des valeurs occidentales, alors qu'en Chine "la recherche d'harmonie, l'oblique, la réconciliation des contraires et la mise sous le boisseau des différences" sont prioritaires.

"Cette recherche questionne aussi la France sur son modèle d'intégration aujourd'hui étriqué et rigide", écrit Richard Beraha. Qu'il s'agisse des Chinois ou d'autres, il paraît inévitable que la société française, aujourd'hui mondialisée, se livre à une introspection en matière d'intégration. La Chine à Paris, qui souhaite "faire confiance à l'avenir, quand beaucoup agitent les spectres du communautarisme souterrain ou de la régression identitaire", y contribue.

M. H.

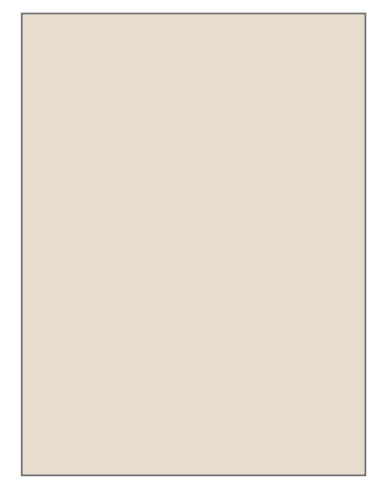

\section{Yannick Torlini}

Nous avons marché

Al Dente 2014, 148 p., $15 €$

"Sur la fine limite entre exister et ne pas exister sur la fine limite du sensible nous avons marché gardé l'équilibre suspendus à un vide plus grand que nous et sans vertige et sans peur sans même imaginer la chute possible et le fracas de nos carcasses en bas tout en bas du devenir nous avons marché et marché et marché encore en équi- libre sur cette fine limite entre le possible et l'impossible nous avons parcouru falaise après falaise toujours attentifs à ce monde qui sous nos enjambées dépérit désespère abandonne nous avons marché toujours car nous ne savons rien faire d'autre et seulement un pas et un pas et un pas et la pierre toujours la pierre qui meurtrit la plante de nos pieds désagrège le reste de cuir de nos chaussures nous avons marché comme seuls les bons à rien savent le faire nous avons marché un pas et un pas et un pas encore nous nous sommes usés jusqu'à la corde et jusqu'au tendon mais sans réticences sans jamais jamais jamais regarder en arrière ni émettre le souhait d'être ail- 
leurs d'être demain d'être hier nous avons marché simplement là simplement engourdis courbatus et sales aussi nous avons marché et marché et marché à pierre fendre dans les jours creux les promesses d'un matin qui n'en finit pas et la curieuse quotidienneté de la pluie sur nos visages travaillés par les ans nous avons marché et il a fait froid très souvent (...)."

Nous avons marché est un recueil de trois textes parmi les plus originaux par son écriture - "ses" écritures plutôt - sur la migration, la mobilité, l'exil, la fuite, l'enfermement... La citation est extraite d'une longue phrase qui court, sans ponctuation, sur une cinquantaine de pages, mue par son propre souffle, sa propre énergie. Dense et pleine, la phrase progresse, gonfle, se renforce, nourrie de scansions, de répétitions, d'émotions, d'images renouvelées, d'un horizon qui va s'élargissant. Il s'agit d'un poème, à lire et à écouter, sur la marche et la décision de partir, sur cette respiration ontologique, indispensable à l'espèce humaine majoritairement sédentaire : la marche, depuis les origines africaines jusqu'à Calais. La marche et la fragilité du migrant, l'instable équilibre du marcheur au-dessus du vide, la transformation des corps et des âmes...

Yannick Torlini poursuit la même expérimentation de la langue et de la musique des mots dans les deux autres textes du recueil, "Tenir registre" et "Tarik (manuel d'exil)". Dans ce dernier, il ajoute des illustrations et des figures quasi enfantines. Ici, la répétition est faite d'entrechoquements, de heurts. Les mots comme les phrases sont refaçonnés, désarticulés, scandés, arrêtés à l'image des corps disloqués, des langues démembrées dans et par l'exil. Les temps se télescopent, les souvenirs et les fantômes tourmentent des âmes fiévreuses. Et pourtant, "le chemin mène toujours quelque part", n'en déplaise à "ceux qui ne savent plus ce qu'est la souffrance".

Dans La Langue et ses monstres (P.O.L., 2014), Christian Prigent évoque la littérature comme "expérience radicale de ce qui nous parle et nous assujettit". Une expérience qui n'aurait d'intérêt "que si ses voix excentriques traversent les représentations couramment admises pour composer de nouveaux accords avec le désir des hommes, leur angoisse, leur sensation d'un monde vivant". L'écriture de Yannick Torlini n'est pas un exercice gratuit et fumeux. Elle est pour le lecteur une expérience intellectuelle et physique, émotionnelle. La poétique du langage, l'écriture indocile et perturbatrice, les assemblages, le jeu des répétitions et des scansions (r)éveillent les sensibilités, stimulent rêves et imaginaires, aident à (re)penser le monde. M. H. 


\section{Minh Tran Huy}

Voyageur malgré lui

Flammarion, 2014

230 p., $18 €$

Comment raconter d'où l'on vient, de quel monde, de quelle culture, de quels silences, sans écrire son autobiographie ? Minh Tran Huy, née en France de parents venus du Viêtnam dans les années 1960, a choisi le détour de la fiction.

Dans Voyageur malgré lui, Line, la narratrice, prend quelques jours de congé à New York et découvre dans un musée l'existence d'Albert Dadas, un ouvrier gazier du XXe siècle qui souffrait de dromomanie ou folie du fugueur. Line, qui aime fréquenter les musées et se concentrer sur autre chose que les bruits du monde qu'elle enregistre pour une agence de création sonore, va se passionner pour ce premier cas de "tourisme pathologique" et se plonger dans l'étude qu'un médecin lui a consacrée. Mais très vite lui reviennent les souvenirs d'autres voyageurs malgré eux: Samia Yusuf Omar, cette jeune sprinteuse somalienne qui a enflammé les Jeux olympiques de Pékin en 2008 avant de périr noyée pour avoir tenté d'émigrer, et aussi des membres de sa propre famille, à commencer par Thin, l'oncle bizarre, qui avait traversé plusieurs océans et continents "tout en rêvant de rentrer chez lui pour n'en plus bouger, et ce désir était si fort, sa détresse si violente, qu'ils en avaient détruit sa raison". Sa mort avait pour la première fois provoqué quelques confidences de son père. Pas étonnant que sa fille rêve de "collectionner les différentes formes de silence". Dans la deuxième partie du livre, alors que Line vole vers la France, consciente que voyager librement est un privilège, se déroule un récit à deux voix de l'histoire familiale. À celle de la jeune femme se mêle celle de son père, qui, alors que la mémoire commençait à le quitter, éprouva soudain une urgence à raconter sa jeunesse dans un pays déchiré par les guerres, contre les Français, puis contre les Américains.

Si sa silhouette élégante, son exigence bienveillante sont présentes dès les premières pages, c'est "son passé de langueur et d'anémie" que l'on découvre ensuite, la rage de réussir d'un petit paysan orphelin du Nord-Viêtnam qui est devenu ingénieur français, le sort tragique de ses compagnons d'études et, plus tard, le constat que partagent nombre d'exilés: "Presque malgré nous, nous commencions de nous établir en ces lieux où nous n'avions pensé que passer. Nous commencions de construire une existence, et même de la prolonger : ta soeur a vu le jour (...) et trois ans après, tu es né." Sans oublier le prix à payer : l'impression de n'être chez soi nulle part, le "double exil", comme l'appelle Abdelmalek Sayad. Le livre refermé, on se prend à penser que Minh Tran Huy a adopté le même cheminement que ce père si discret, si pudique, en le révélant lentement - n'a-t-il pas mis plus de deux décennies à parler de lui ? On ne peut qu'être ému par cette constellation de destins dont il risquait de ne rien rester. Le roman se dresse contre la fatalité de l'effacement, s'interroge de façon délicate sur la transmission et révèle toute la richesse des identités faites d'un mélange de langues et de cultures. Élisabeth Lesne 


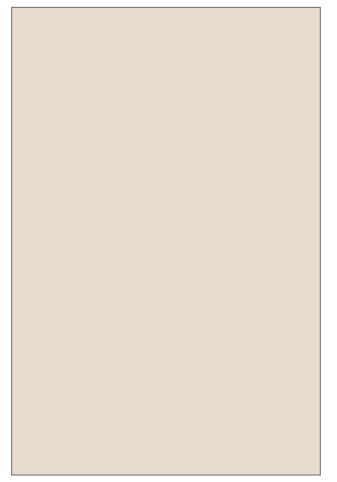

\section{Valérie Zenatti}

Jacob, Jacob

L'Olivier, 2014,

168 p., $16 €$

Sur la couverture du livre, laphotod'unjeune homme en uniforme nous regarde, vareuse ouverte, béret de travers, regard clair et profond, c'est Jacob.

Dans ce roman, la traductrice d'Aharon Appelfeld et l'auteure d'Une bouteille dans la mer de Gaza se tourne vers la terre d'origine de ses parents, I'Algérie coloniale, pour suivre le court destin de ce grand-oncle dont la photo l'attirait dans l'album de famille. "Il est beau Jacob, même de dos", dit Lucette, sa voisine amoureuse, en le regardant partir pour l'armée. Nous sommes en juin 44 et Joseph Mekli, le petit dernier d'une famille juive de Constantine, vient d'être enrôlé, "défenseur d'une Europe qui avait tué ou laissé mourir ses juifs". Jacob occupe une place particulière dans cette famille pauvre. Non seulement il est le seul à avoir passé le bac, mais, dans un monde archaïque d'hommes frustres et violents, sa gentillesse, sa faculté à être aimé de tous, son humour aussi, le rangent du côté des femmes et des enfants.

Après quelques semaines d'entraînement dans le Hoggar, où la disposition régulière des tentes "lui serre le coeur, on dirait des tombes dans le carré d'un cimetière", c'est le débarquement en Provence, au sein de la $3^{e}$ division algérienne, où se retrouvent mêlés, combattant côte à côte, musulmans, juifs et Fran- çais d'Algérie. Cette armée victorieuse de libération, on la suit jusqu'en Alsace auprès de Jacob et de ses camarades. Valérie Zenatti excelle à décrire cette campagne dans une langue charnelle et sensuelle qui englobe tout : sensations et réflexions, exaltations et appréhensions, descriptions et conversations, premières et dernières fois. L'eau et la musique, éléments favoris de Jacob, caractérisent aussi le style de Valérie.

Vient ensuite le temps du deuil - des quatre, seul rentre Ouabedssalam, blessé au champ d'honneur, mais jamais décoré. De Jacob, d'une vie si courte - dix-neuf ans -, que reste-t-il ? Une photo, des souvenirs qui s'estomperont, son prénom, comme en écho.

Jacob a été le premier à découvrir une France qu'il ne connaissait qu'à travers les livres. Quelque vingt ans plus tard, ce sera au tour de sa famille. L'assassinat de Cheik Raymond, la star de la musique arabo-andalouse adulée par les juifs et les Arabes, "était plus qu'un avertissement : un signe funeste. Partez, partez, nous avons choisi de rompre le lien qui nous unissait depuis des siècles. Vous continuez à parler notre langue, à vous enduire les mains de henné pour les fiançailles de vos enfants, à cuisiner comme nous, mais vous êtes des traîtres, alliés depuis cent ans aux français, remplis d'orgueil à la pensée de posséder leurs cartes d'identité grises".

Dans ce roman nourri de souvenirs familiaux, la fiction, la tendresse et l'émotion ont aussi toute leur place. E. L. 